\title{
Apple Internal Quality Inspection Using Hyperspectral Image Technology
}

\author{
Xiao-Yan Chen \\ College of Mechanical and Electrical Engineering, \\ Sichuan Agriculture University \\ Ya'an city,Sichuan Province 625000, China
}

Wen-Tao Chen

College of Mechanical and Electrical Engineering, Sichuan Agriculture University

Ya'an city,Sichuan Province 625000, China

\section{Jia-Sui Lv}

College of Mechanical and Electrical Engineering, Sichuan Agriculture University

Ya'an city,Sichuan Province 625000, China

\begin{abstract}
The internal parameters are important indexes for detecting the quality of the apples. This paper extracted spectral values of the apples from $400-1000 \mathrm{~nm}$ with the hyperspectral image technology, carried out pre-treatment to original spectrums with MSC, performed regression analysis on spectral reflectivity of sugar content and firmness, and finally established prediction model of apple sugar content and firmness with BP (back propagation) artificial neural network. The results show that the correlation coefficient of the prediction model for sugar content is 0.9861 , the average error is $0^{\circ 118}$ Brix; the correlation coefficient of the prediction model for firmness is 0.9771 , the average error is $0.054 \mathrm{Kg} / \mathrm{cm}^{\wedge} 2$. Therefore, it is feasible to detect the internal quality parameter of apples using hyperspectral technology.
\end{abstract}

Keywords-Hyperspectral Images, Sugar Content, Firmness, Artificial Neural Network

\section{INTRODUCTION}

The annual apple yield of China, as a large apple producing country, ranks top on the world.As a result of the backwardness of post-harvest inspection and grading technology, China's apples lack of competitiveness on international apple market and the proportion of export takes up no more than $1.5 \%$ of total yield [1]. Therefore, study on apple quality inspection and rapid and efficient grading is quite necessary. As the living standard is continuously improving, people concern more about internal quality of the apples such as firmness and sugar content. Traditionally, the sugar content and firmness of the apples are measured with precision instruments while the apples are juiced or sliced, i.e. damaging the integrity of the samples, so a kind of rapid and nondestructive method for inspecting the internal quality of the apples is very important.

\author{
Xiang Long \\ College of Mechanical and Electrical Engineering, \\ Sichuan Agriculture University \\ Ya'an city,Sichuan Province 625000, China
Tao Pang
College of Mechanical and Electrical Engineering, Sichuan Agriculture University Ya'an city,Sichuan Province 625000,China scndcxy@163.com

As a rising contactless inspection technology, the hyperspectral image technology integrates the traditional spectral technology and 2D imaging technology and realizes rapid nondestructive inspection of fruits by extracting and analyzing the spectral information and image information of the samples to be inspected [2]. Over the past few years, the hyperspectral image technology is widely applied in agricultural product quality inspection and grading [3-7]. E Masry et. al. [8] Inspecting internal water content and soluble solid content of strawberry with the hyperspectral image technology whose wave length is ranging from $400 \sim 1000 \mathrm{~nm}$ and establishing prediction models; Tian Youwen et. al. [9] Correct characteristic vectors of apple insect damages are extracted with the hyperspectral image technology; Guo Enyou et. al. [10] Inspecting soluble solid content of the navel oranges with the hyperspectral image technology whose wave length is ranging from 700 1000nm; Zhao Jiewen, et. al. [11] Inspecting the firmness of the apples with the hyperspectral image technology and multivariate calibration methods.

This paper carried out inspection research on multiple internal parameters of the apple with the hyperspectral image technology, searched characteristic wave bands that can accurately inspect the internal parameters of the apples through relevant image and data processing methods, and finally utilize BP artificial network to carry out modeling predictive analysis to the characteristic wave bands so as to provide theoretical basis for realizing online apple internal quality inspection and grading in days to come.

\section{Test MATERIALS AND EQUiPMENT}

\section{A. Test Materials}

80 apples (Red Fuji) with appropriate sizes, shapes, normal skin color and without noticeable scars were selected as test materials. In order to avoid influences of uneven lighting and outdoor temperature on local sugar content and spectral reflectance, the 80 samples were 
sealed with plastic bags and placed in $4^{\circ} \mathrm{C}$ environment for storing $24 \mathrm{~h}$.Before the test was carried out, the apples were taken out of the storage environment and placed in normal environment for $12 \mathrm{~h}$, and then the hyperspectral images were acquired.

\section{B. The Hyperspectral System and Image Acquisition}

The hyperspectral system adopts Beijing Zolix Instrument Co., Ltd.'s GaiaSorter hyperspectral sorter. Fig.1shows the structure of the hyperspectral system.This system mainly consists of light source, 1344 pixels $* 1024$ pixels CCD spectral camera, motorized movable platform, computer and control software SpecView. The spectral range of the hyperspectral camera is $400-1000 \mathrm{~nm}$, the spectral resolution is $2.8 \mathrm{~nm}$, and the width of the incident light slit is $30 \mu \mathrm{m}$. The light source are 2 sets of brominetungsten lamps (rated power is 200W), the lights generated by thermal radiation of the light source were irradiated on the sample bench. The light source unevenness within the cubic space of $300 * 20 * 100 \mathrm{~mm}(\mathrm{~L} * \mathrm{~W} * \mathrm{H})$ is within $5 \%$.

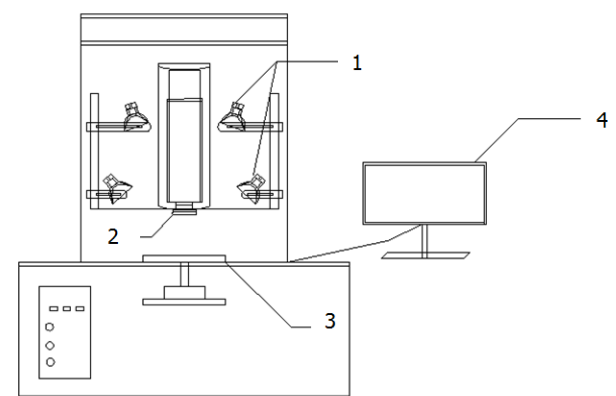

1.Light source 2.CCD Camera 3.Displacement platform controller 4.Computer

Figure 1. Schematic diagram of the hyperspectral image system

\section{DATA ACQUisition AND PROCESSING}

\section{A. Acquisition and Pre-processing of Hyperspectral Images}

At the time of acquisition, the lens was $16 \mathrm{~cm}$ above the surface of the apples. In acquisition of hyperspectral images, the exposure time was set as $11 \mathrm{~ms}$ in SpecView and the moving speed of the platform was $0.40 \mathrm{~cm} / \mathrm{s}$. By irradiating the sample apples on the motorized moving platform, the reflected light of the samples were acquired by CCD spectral camera and one-dimensional images and spectral information were obtained. With the motorized moving platform carries the samples to operate continuously, a continuous one dimensional images and real-time spectral information were obtained. All data were recorded by the software and a three-dimensional cube containing image information and spectral information was finally obtained.

In order to minimize the influences of background noise and quantum efficiency of camera, ENVI 5.1 was used to carry out black and white correction to original spectrums.Keep the parameters of the image acquisition system unchanged, carry out sample white image $\mathrm{W}$ to standard white board with $99 \%$ reflectivity, and then screw on the lens cap to carry out hyperspectral imaging and obtain total black spectrum $\mathrm{B}$, the correction calculation formula is:

$$
\mathrm{R}=\frac{I-B}{W-B}
$$

Where, $\mathrm{R}$ is the corrected hyperspectral images, $\mathrm{I}$ is original hyperspectral image, $\mathrm{B}$ is black image, $\mathrm{W}$ is the diffuse reflection image.Fig. 2 shows the spectra of the relative reflectance of apples.

\section{B. Inspection of sugar content of the samples}

The sugar content of the apples was measured with a LH-T32 portable saccharimeter (Hangzhou Lohand Biological Co.,Ltd,China). The measuring range of this saccharimeter is $0-32^{\circ} \mathrm{Brix}$, the distinguishability is $0.2^{\circ}$ Brix, and it has the temperature automatic compensation function. The detailed measuring method is that taking 4 small pieces of pulp from front, rear, left and right sides of an apple sample, respectively mesh them into juice and dripped on deviation prism of the saccharimeter, directly read the values and calculate the mean value of four times, and then the sugar content of an apple sample.

\section{Practical measurement of sample firmness}

The firmness of apples was measured with the GY-4 fruit firmness tester (Beijing THY Science \& Technology Co., Ltd, China). The scale indication of this firmness tester is $2-15 \mathrm{~kg} / \mathrm{cm}^{\wedge} 2(\times 105 \mathrm{pa})$, the probe size is $\Phi 3.5 \mathrm{~mm}$ and the division value is $0.1 \mathrm{~kg} / \mathrm{cm}^{\wedge} 2(\times 105 \mathrm{pa})$. The detailed calculation formula is $\mathrm{P}=\mathrm{N} / \mathrm{S}$, of which $\mathrm{S}$ is the stress area of the tested fruit, $\mathrm{N}$ is the pressure of dynamometer spring borne by the fruit and $\mathrm{P}$ is the firmness value of the fruit. At the time of measurement, the probe of the durometer is perpendicular to the area of apple to be tested and inserted into the apple at an even speed till the probe reaches to the scale mark $(10 \mathrm{~mm})$. While the probe is inserting, the indicator rotates and the reading that the indicator points to when it stops is the tested firmness of the apple. Average the firmness value of three times to get the firmness of an apple sample.

\section{Test Results And AnAlysis}

\section{A. Linearization of Reflectance Spectrum}

The apple sample spectral curve can be obtained from the hyperspectral images that are collected and pre-treated, as shown in Fig.2. Due to influences of light wave scattering and shifting, it is difficult to obtain reflective characteristic point of spectrum and the corresponding wavelength. In order to eliminate the influences of light wave scattering and shifting, MSC (Multiplicative Scatter Correction) is utilized to process the spectrum. The MSC method is a frequently used data processing method in present multi-wavelength calibration modeling. The spectral data obtained after scatter correction can effectively eliminate the influences of scattering including correction of base line translation and shifting which 
enhances spectral absorption information related to component content. The algorithm is as below:

At first, calculate the average spectral value of all samples:

$$
\overline{\mathrm{A}_{\mathrm{j}}}=\sum_{\mathrm{i}=1}^{\mathrm{n}} \mathrm{A}_{\mathrm{i}, \mathrm{j}} / \mathrm{n}
$$

Second, carry out unary linear regression to the spectral value of each sample and the average spectral value to obtain regression coefficient $\mathrm{mi}$ and bi:

$$
\mathrm{A}_{\mathrm{i}}=\mathrm{m}_{\mathrm{i}} \overline{\mathrm{A}}+\mathrm{b}_{\mathrm{i}}
$$

Finally, Multiplicative Scatter Correction:

$$
A_{i(M S C)}=\frac{\left(A_{i}-b_{i}\right)}{m_{i}}
$$

Where $i=1,2 \ldots \ldots . n, n$ - sample quantity, $j$ - refers to the jth wave number, Aij - spectral value of the sample.

Fig. 3 shows the spectra of the relative reflectance of apples after MSC. Comparing to Fig. 2, it can see the spectral reflective characteristic points and corresponding wavelength more clearly in Fig.3.

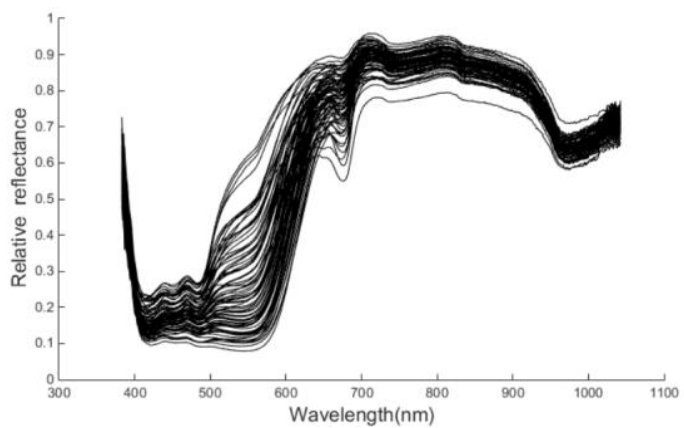

Figure 2. Relative reflectance spectral

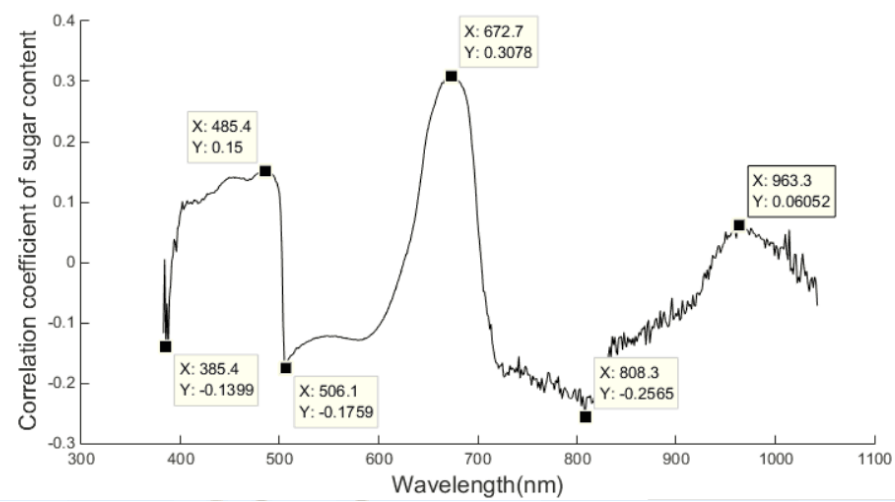

Figure 4. Correlation analysis on sugar content and spectral reflectivity values

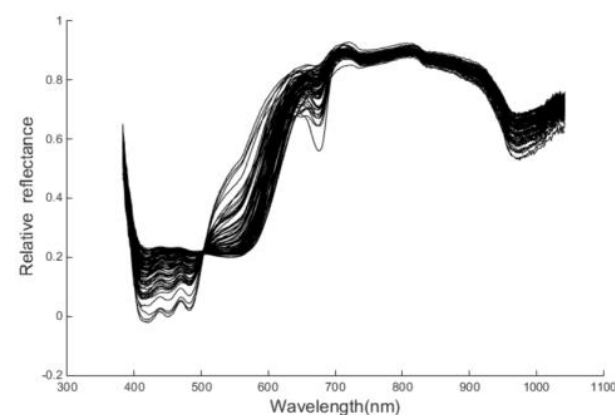

Figure 3. Relative reflectance spectral after MSC

\section{B. Regression Analysis of Sugar Content, Firmness and Reflected Spectral Values}

In order to select characteristic wave band values that are related to sugar content and firmness, we respectively established relevant analyses on sugar content and firmness with spectral regression curve with Pearson Correlation Coefficient Method. Fig. 4 and Fig. 5 are relevant coefficient diagrams of the sugar content and firmness with the spectral reflectivity values. On the basis of the principle of local maximum value and minimum value, the six wavelengths were respectively selected as characteristic wave bands of the sugar content and firmness, and Table 1 lists the selected characteristic wave bands of sugar content and firmness. 


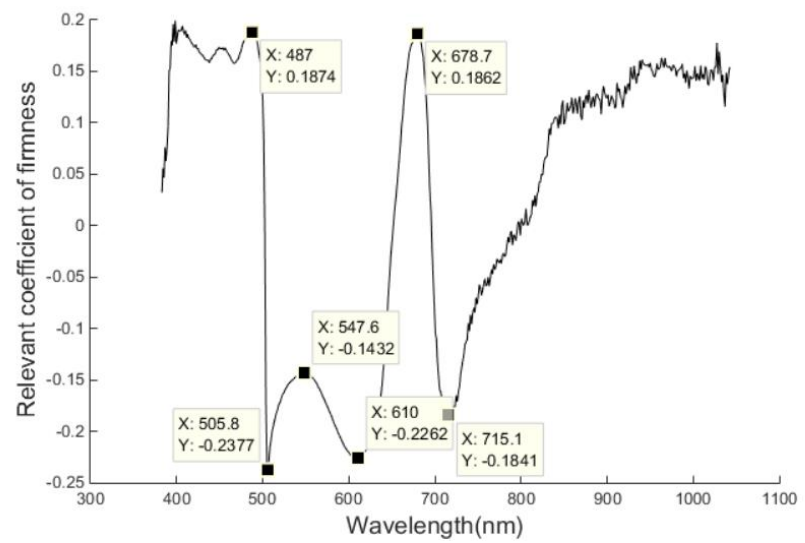

Figure 5. Correlation analysis on firmness and spectral reflectivity value

TABLE 1. PREDICTION CHARACTERISTIC WAVE BANDS OF SUGAR CONTENT AND FIRMNESS

\begin{tabular}{lcccccc}
\hline $\begin{array}{l}\text { Internal } \\
\text { parameter }\end{array}$ & \multicolumn{6}{c}{ Characteristic wave band (nm) } \\
\hline Sugar content & 385.4 & & & 672.7 & & \\
& & 485.4 & 506.1 & & 808.3 & 963.3 \\
Firmness & 487 & & & & & 715.1 \\
& & 508.8 & 547.6 & 610 & 678.7 & \\
\hline
\end{tabular}

\section{BP Artificial Neural Network Prediction Model}

BP artificial neural network is a supervised study algorithm of forward neural network, is multi-layer nonfeedback connecting network consisting of input layer, output layer and several intermediate hidden layers. The main characteristic is that the signals are forward transmitted and the errors are backward transmitted. We established prediction model of sugar content and firmness by utilizing BP artificial neural network. This network consists of 1 input layer, 2 hidden layers and 1 output layer. The input layer comprises of 6 nodes, the input is the 6 selected characteristic wave bands respectively corresponded to sugar content and firmness; the double hidden layers respectively comprise of 3 and 2 nodes, and the output layer comprises of 1 node which is the sugar content or the firmness. The neural network transfer functions are radbas, logsig and purelin, the training function is trainlm. In test, $3 / 4$ samples $(60$ pcs $)$ were used as training set for modeling, and the rest 1/4 (20 pcs) were used as prediction set for verification.

Fig.6 is the training results of sugar content and firmness, and it is known from the figure that the correlation coefficient of sugar content training result $\mathrm{R}^{\wedge} 2$ is 0.9742 , the RMSEC is 0.9848; the correlation coefficient of firmness training result $\mathrm{R}^{\wedge} 2$ is 0.9613 , the RMSEC is 0.2508 .

Fig.7 is the prediction results of sugar content and firmness, and it is known from the figure that the correlation coefficient of sugar content prediction result $\mathrm{R}^{\wedge} 2$ is 0.9861 , the RMSEP is 1.3070; the correlation coefficient of firmness prediction result $\mathrm{R}^{\wedge} 2$ is 0.9771 , the RMSEP is 0.5460. Table 2 shows neural network training and prediction parameters. The correlation coefficient of prediction result of sugar content $\mathrm{R}^{\wedge} 2$ is 0.9861 , RMSEP is 1.3070. The correlation coefficient of the firmness prediction result $\mathrm{R}^{\wedge} 2$ is 0.9771 , RMSEP is 0.5460 .
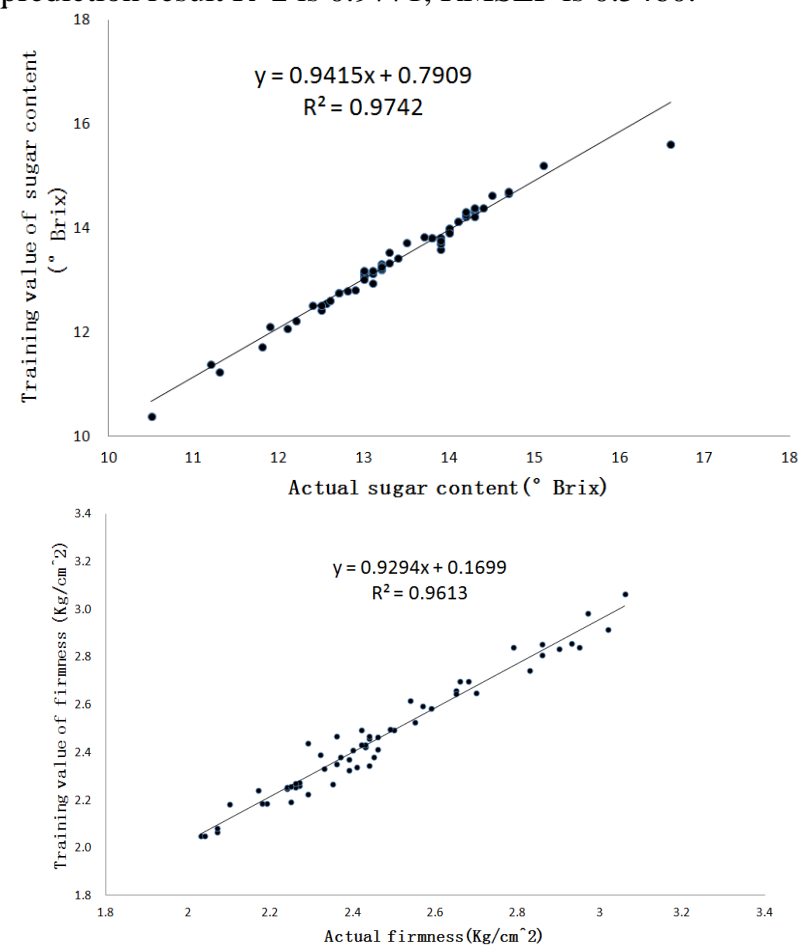

Figure 6. Training result of the training set. 

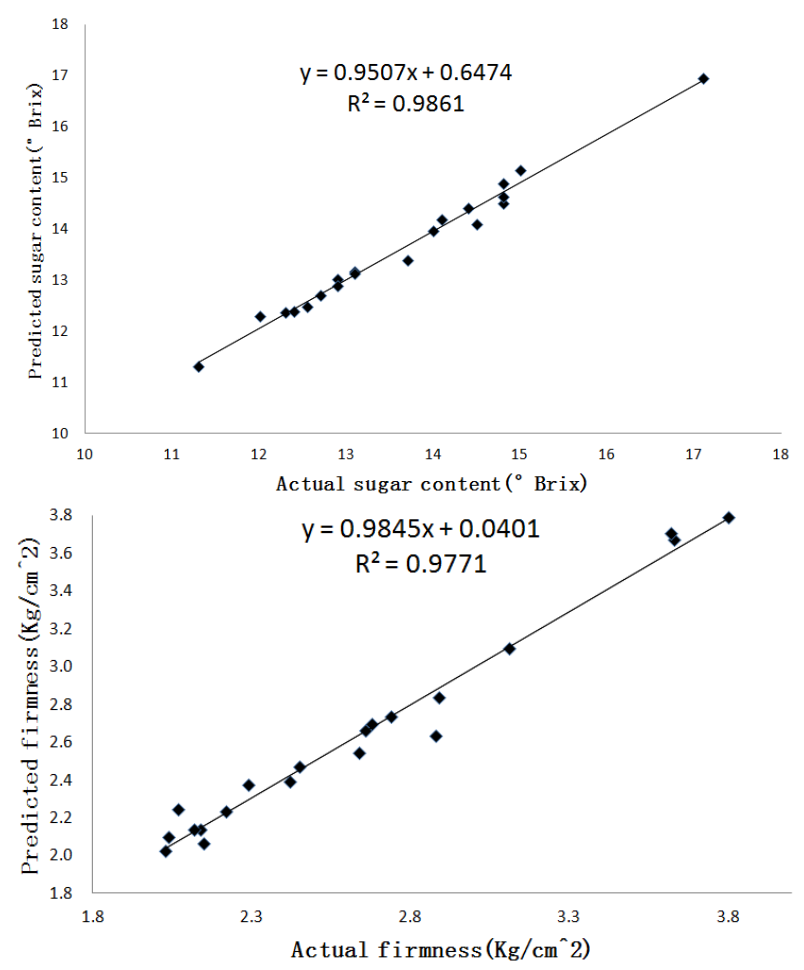

Figure 7. Verification result of the prediction set
TABLE 2. NEURAL NETWORK TRAINING AND PREDICTION PARAMETERS

\begin{tabular}{lcccc}
\hline parameter & \multicolumn{2}{c}{ Training set (60 samples) } & \multicolumn{2}{c}{ Prediction set (20 samples) } \\
& $\mathrm{R}^{\wedge} 2$ & $\mathrm{RMSEC}$ & $\mathrm{R}^{\wedge} 2$ & RMSEP \\
\hline Sugar content & 0.9742 & 0.9848 & 0.9861 & 1.3070 \\
Firmness & 0.9613 & 0.2508 & 0.9771 & 0.5460 \\
\hline
\end{tabular}

Table 3 shows the prediction verification data of the prediction set. The result shows that the maximum error of the sugar content is $0.399^{\circ} \mathrm{Brix}$, the minimum is $0.006^{\circ}$ Brix, and the average is $0.118{ }^{\circ}$ Brix. The maximum error of the firmness is $0.246 \mathrm{Kg} / \mathrm{cm}^{\wedge} 2$, the minimum error is $0.001 \mathrm{Kg} / \mathrm{cm}^{\wedge} 2$, and the average error is $0.054 \mathrm{Kg} / \mathrm{cm}^{\wedge} 2$. The above conclusion shows that the hyperspectral image technology can accurately inspect the sugar content and firmness of the apples, so it is feasible that this technology is utilized for non-destructive inspection of the apples. 
TABLE 3. DATA OF SUGAR CONTENT AND FIRMNESS PREDICTED BY NEURAL NETWORK

\begin{tabular}{|c|c|c|c|c|c|c|}
\hline \multirow[b]{2}{*}{$\begin{array}{c}\text { Prediction } \\
\text { Sample }\end{array}$} & \multicolumn{3}{|c|}{ Sugar content } & \multicolumn{3}{|c|}{ Firmness } \\
\hline & $\begin{array}{c}\text { Measured } \\
\text { values } \\
{ }^{\circ} \text { Brix }\end{array}$ & $\begin{array}{c}\text { Prediction } \\
\text { value } \\
{ }^{\circ} \text { Brix }\end{array}$ & $\begin{array}{l}\text { Error } \\
{ }^{\circ} \text { Brix }\end{array}$ & $\begin{array}{c}\text { Measured } \\
\text { value } \\
\mathrm{Kg} / \mathrm{cm}^{\wedge} 2\end{array}$ & $\begin{array}{c}\text { Prediction } \\
\text { value } \\
\mathrm{Kg} / \mathrm{cm}^{\wedge} 2\end{array}$ & $\begin{array}{c}\text { Error } \\
\mathrm{Kg} / \mathrm{cm}^{\wedge} 2\end{array}$ \\
\hline 1 & 14.100 & 14.192 & 0.092 & 2.15 & 2.064 & 0.086 \\
\hline 2 & 13.700 & 13.401 & 0.299 & 2.64 & 2.543 & 0.097 \\
\hline 3 & 14.800 & 14.892 & 0.092 & 2.88 & 2.634 & 0.246 \\
\hline 4 & 15.000 & 15.153 & 0.153 & 3.63 & 3.676 & 0.046 \\
\hline 5 & 13.100 & 13.162 & 0.062 & 2.14 & 2.139 & 0.001 \\
\hline 6 & 14.800 & 14.626 & 0.174 & 2.89 & 2.840 & 0.050 \\
\hline 7 & 12.300 & 12.380 & 0.080 & 2.29 & 2.374 & 0.084 \\
\hline 8 & 12.000 & 12.291 & 0.291 & 2.12 & 2.138 & 0.018 \\
\hline 9 & 11.300 & 11.308 & 0.008 & 2.22 & 2.235 & 0.015 \\
\hline 10 & 14.500 & 14.101 & 0.399 & 2.45 & 2.473 & 0.023 \\
\hline 11 & 12.900 & 13.020 & 0.120 & 2.04 & 2.099 & 0.059 \\
\hline 12 & 12.900 & 12.886 & 0.014 & 2.74 & 2.734 & 0.006 \\
\hline 13 & 14.000 & 13.967 & 0.033 & 2.66 & 2.662 & 0.002 \\
\hline 14 & 17.100 & 16.958 & 0.142 & 3.62 & 3.707 & 0.087 \\
\hline 15 & 12.700 & 12.714 & 0.014 & 2.68 & 2.698 & 0.018 \\
\hline 16 & 12.400 & 12.390 & 0.010 & 2.42 & 2.390 & 0.030 \\
\hline 17 & 14.800 & 14.512 & 0.288 & 3.11 & 3.099 & 0.011 \\
\hline 18 & 14.400 & 14.406 & 0.006 & 3.80 & 3.790 & 0.010 \\
\hline 19 & 12.550 & 12.491 & 0.059 & 2.07 & 2.246 & 0.176 \\
\hline 20 & 13.100 & 13.123 & 0.023 & 2.03 & 2.024 & 0.006 \\
\hline average & 13.623 & 13.599 & 0.118 & 2.629 & 2.628 & 0.054 \\
\hline
\end{tabular}

\section{CONCLUSION}

It tested utilizing the hyperspectral image technology to inspect the internal parameters of the apples, i.e. sugar content and firmness, within the band range of $400-$ $1000 \mathrm{~nm}$. The original spectrum was pre-processed with MSC, regression analysis was carried out to sugar content and firmness with spectral reflectivity amount for selecting 6 relevant characteristic wave bands, and such 6 bands were utilized for carrying out neural network modeling and prediction analysis. The correlation coefficient of prediction result of sugar content $\mathrm{R}^{\wedge} 2$ is 0.9861 , RMSEP is 1.3070 , the maximum sugar content error is $0.399^{\circ} \mathrm{Brix}$, the minimum is $0.006^{\circ}$ Brix, and the average error is $0.118{ }^{\circ}$ Brix. The correlation coefficient of the firmness prediction result $\mathrm{R}^{\wedge} 2$ is 0.9771 , RMSEP is 0.5460 , the maximum error of the firmness is $0.246 \mathrm{Kg} / \mathrm{cm}^{\wedge} 2$, the minimum error is $0.001 \mathrm{Kg} / \mathrm{cm}^{\wedge} 2$, and the average error is $0.054 \mathrm{Kg} / \mathrm{cm}^{\wedge} 2$. Therefore, it is feasible that the hyperspectral image technology is applied for inspection of internal quality of the apple.

In future research, we will increased sample quantity of prediction models, carry out deeper study, perfect prediction model so as to provide theoretical basis for realizing detection of online internal parameters of the apples.

\section{ACKNOWLEDGMENTS}

This wok was funded by the Natural Science of Sichuan Provincial Education Department (grant no. 15ZA0019).

\section{REFERENCES}

[1] The Ministry of Agriculture. The international apple trade situation and China apple export situation of $[\mathrm{J}]$. Chinese Fruit Industry Information, 2006(4): 6-8. (in Chinese)

[2] Nondestructive inspect of apple quality with hyperspectral imaging[J].Infrared and Laser Engineering, 2014,43(4):1272-1277.

[3] Lorente D, Aleixos N, et a1. Recent advances and applications of hyperspectral imaging for fruit and vegetable quality assessment[J]. Food Bioprocess Technol, 2012, 5(4): 1121-1142.

[4] Elmasry G, Kamruzzaman M , Sun D W,et a1.Principles and applications of hyperspectral imaging in quality evaluation of agrofood products: A review [J]. Critical Reviews in Food Science and Nutrition, 2012, 52(11): 999-1023.

[5] Li Jiang bo, Rao Xiuqin ,Ying Yibin. Advance on application of hyperspectral imaging to nondestructive detection of agricultural products external quality [J], Spectroscopy and Spectral Analysis, 2011,31(8): 2021 — 2026. (in Chinese with English abstract) 
[6] Hong Tiansheng, Qiao Jun ,Ning Wang, et a1. Non-destructive inspection of Chinese pear quality based on hyperspectral imaging technique[J].Transactions of the Chinese Society of Agricultural Engineering, 2007, 23(2):151-155.(in Chinese)

[7] WU Longguo,HE Jianguo, HE Xiaoguang, et al. Research progress of hyperspectral imaging technology in non-destructive detection of fruit[J]. LASER \& INFRARED, 2013,43(9):990-996.

[8] Masry E G. Hyperspectral imaging for nondestructive deterruination of some quality at tributes for strawberry[J].Journal of Food Engineering,2007,81:98-107.

[9] Tian Youwen, Cheng Yi, Wang Xiaoqi, et a1. Feature vectors determination for pest detection on apples based on hyperspectral
imaging[J]. Transactions of $\mathrm{t} h$ e Chinese Society of Agricultural Engineering (Transactions of the CSAE), 2014, 30(12): 132-139

[10] Guo Enyou , Liu Muhua, Zhao Jiewen, et a1 . Nondestructive detection of sugar content on Navel orange with hyperspectral imaging [J]. Transactions of the CSAM ,2008,39(5): 91—93,103. (in Chinese)

[11] Zhao Jiewen, Chen Quansheng, Saritporn Vittayapadung, et al. Determination of apple firmness using hyperspectral imaging technique and multivariate calibrations[J].Transactions of the CSAE, 2009, 25(11):226 - 231.(in English) 